\title{
Combatting rickets
}

\section{Researchers, nutritionists and policy-makers face a problem over rickets in Britain, as Alastair Hay explains}

A QUICK survey of the British public would suggest that its nutritional problems are not due to a lack of food. Yet even in the midst of overconsumption a specific nutritional deficiency-that of vitamin D-has been defined in certain sections of the population. Rickets, known to generations of Europeans as the 'English disease' is now a problem in the children of Britain's Asian community and occurs sporadically in some of the infants of the country's poorest populations. It was first reported as being a serious problem in the Asian children of Glasgow almost sixteen years ago and has since been observed in one Asian community after another throughout the country.

The summer issue of Science for People - the publication of the British Society for Social Responsibility in Science (BSSRS)-asserts that the UK Department of Health and Social Security (DHSS) actually causes rickets. The journal is noted for a blunt approach to the issues it tackles, but this attack will not be viewed altogether unfavourably by many clinicians concerned with the health of Britain's Asian population and particularly with the problem of rickets. They point out that the problem is well known-perhaps too well known -and that there has been no shortage of surveys and quotable figures. They claim that most of the answers to this proven dietary deficiency are known, but that the DHSS has so far failed to adopt a positive approach.

Faced with such hostility the DHSS has been giving the matter serious attention. The department's Committee on Medical Aspects of Food Policy (COMA) is reviewing the whole question of bone disease contracted as a result of nutritional deficiency. The committee's deliberations have been somewhat protracted and are still by no means completed. But a report is expected that will produce some recommendations for action.

It is children who suffer from rickets; the same bone disorder in adults is termed osteomalacia. It results from an inadequate supply of vitamin D. The vitamin is required for the active absorption of calcium and phosphorus from the gut. When this mineral supply is reduced in vitamin $\mathrm{D}$ deficiency, the body, in order to maintain blood mineral levels, has recourse to its only other source of supply, the bones. Bone growth ceases, the skeletal structure become progressively weaker and eventually deteriorates to produce 'bow-leg' or 'knock-knee' rickets.

Although biochemists are having a field day trying to elucidate vitamin D's exact mode of action, at the nutritional level the problem would seem to be simply that of ensuring an adequate supply. But the solution is not as easy as it seems, which could be one explanation why the DHSS is taking so long to formulate a policy. The question that needs answering concerns the most effective vehicle for administering the vitamin: should it be done through chapati flour, or milk, or vitamin $\mathrm{D}$ capsules, or tablets?

As a major constituent of the diet

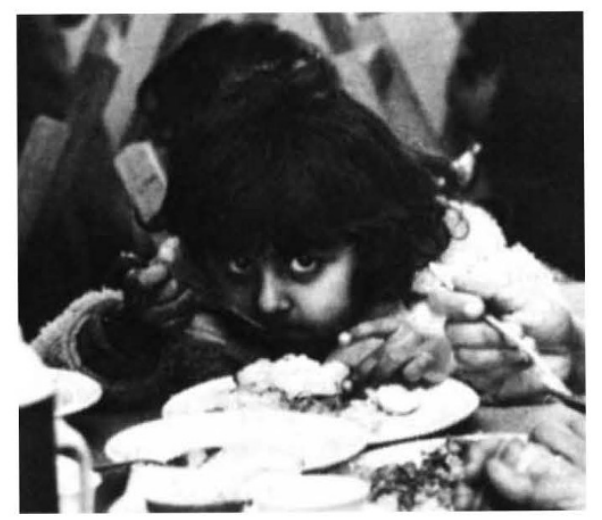

of most Asian families, chapati flour would seem to be an ideal medium through which to add extra vitamin $\mathrm{D}$ to the diet. The technical problems of mixing the vitamin and of its preservation have been solved, and clinical trials using vitamin D-fortified chapati flour have shown its effectiveness as a method of administering the vitamin. A problem may arise with regard to the concentration gradient. It is children, not adults, who are most in need of extra vitamin $D$, and adults consume more flour per head. The answer would be to have two types of flour, one fortified and recommended for children, the other unfortified. But as a spokesman for a bread company says: "Someone has to pay for the process".

Milk poses different problems. There are no legal constraints to forbid fortifying chapati or brown flour with nutrients; this is not true of milk. Britain is bound by an EEC directive forbidding any alteration to the composition of milk. When the issue was discussed in Brussels, several European countries were in favour of legislating for a $3 \frac{1}{2} \%$ fat level. Britain fought this, primarily on financial grounds, and insisted on the right to have milk virtually unaltered from cow to con- sumer (most milk would have to have fat removed to attain the $3 \frac{1}{2} \%$ figure). The British case was conceded, but at the time Asian rickets was not considered to be a problem; now it is. Before embarking on any milk fortification programme Britain would first have to return to the negotiating table, which could be embarrassing, but little choice may remain if the DHSS comes out in favour of using vitamin Dfortified milk. The arguments in favour of its use are strong. As children drink more milk than adults, it would reach the population most in need of treatment. Those in favour of milk point out that Canada fortifies milk, milk products and margarine with vitamin D; the United States does the same and much else besides.

As for the third suggestion that vitamin $\mathrm{D}$ capsules or tablets be dispensed either at clinics or in schools, staff in some schools are opposed on the grounds that the procedure of identifying children in need from the school register is too complicated.

As breast-fed babies in Britain rarely develop rickets any health education policy ought to discuss the merits of breast feeding as well. The reason for this immunity is not yet clear, but is probably related to the fact that the breast milk of vitamin D-replete mothers contains a fairly high concentration of the vitamin present as vitamin D sulphate. Some groups of immigrant women stop breast feeding when they arrive in the country and resort to doorstep milk.

\section{No significant impact}

It is generally acknowledged that the health education programmes dealing with rickets have failed to make a significant impact in the Asian community. Some clinicians argue that these programmes have failed dismally in alerting local doctors and health workers to the seriousness of the problem. They add that there is great inertia at the level of the community physician, that many doctors are reluctant to become involved in preventative campaigns, and that many health workers have not been informed that the problem exists.

That rickets is present in the Asian community, and a serious problem, is not in doubt. Some may query the figures for the prevalence of the disease, and argue for more information. Others will say the evidence is now so overwhelming that action must be taken. The DHSS recognises that many doctors expect its COMA committee to make some positive recommendations stressing the need for a food fortification programme backed up by a good health education policy reaching all the Asian community. 